\title{
Bianchi Types II, VIII, and IX String Cosmological Models with Bulk Viscosity in a Theory of Gravitation
}

\author{
V. U. M. Rao, K. V. S. Sireesha, and M. Vijaya Santhi \\ Department of Applied Mathematics, Andhra University, Visakhapatnam 530003, India \\ Correspondence should be addressed to V. U. M. Rao, umrao57@hotmail.com
}

Received 24 November 2011; Accepted 25 December 2011

Academic Editor: W.-H. Steeb

Copyright $@ 2012$ V. U. M. Rao et al. This is an open access article distributed under the Creative Commons Attribution License, which permits unrestricted use, distribution, and reproduction in any medium, provided the original work is properly cited.

We have obtained and presented spatially homogeneous Bianchi types II, VIII, and IX string cosmological models with bulk viscosity in a theory of gravitation proposed by Sen (1957) based on Lyra (1951) geometry. It is observed that only vacuum cosmological model exists in case of Bianchi type IX universe. Some physical and geometrical properties of the models are also discussed.

\section{Introduction}

Lyra [1] proposed a modification to Riemannian geometry by introducing an additional gauge function into the structure less manifold, as a result of which the cosmological constant arises naturally from the geometry. This bears a remarkable resemblance to Weyl's [2] geometry. In subsequent investigations Sen [3] and Sen and Dunn [4] formulated a new scalartensor theory of gravitation and constructed an analog of Einstein's field equations based on Lyra's geometry. Halford [5] has shown that the scalar-tensor treatment based on Lyra's geometry predicts the same effects as in general relativity.

The field equations in normal gauge in Lyra's manifold as obtained by Sen [3] are

$$
R_{i j}-\frac{1}{2} R g_{i j}+\frac{3}{2} \phi_{i} \phi_{j}-\frac{3}{4} g_{i j} \phi_{k} \phi^{k}=T_{i j}
$$

where $T_{i j}$ is the stress energy tensor of the matter, $\phi_{i}$ is the displacement field, and other symbols have their usual meaning as in Riemannian geometry. The displacement field $\phi_{i}$ can be written as $\phi_{i}=(0,0,0, \beta(t))$. 
The study of string theory has received considerable attention in cosmology. Cosmic strings are important in the early stages of evolution of the universe before the particle creation. Cosmic strings are one-dimensional topological defects associated with spontaneous symmetry breaking whose plausible production site is cosmological phase transitions in the early universe. Letelier [6], Krori et al. [7], Mahanta and Mukheriee [8], and Bhattacharjee and Baruah [9] have studied several aspects of string cosmological models in general relativity. Reddy and Rao [10] have studied axially symmetric cosmic strings and domain walls in a scalar tensor theory proposed by sen [3] based on Lyra [1] geometry. Mohanty and Mahanta [11] have studied five-dimensional axially symmetric string cosmological model in Lyra [1] manifold. Rao and Vinutha [12] have studied axially symmetric cosmological models in a scalar tensor theory of gravitation based on Lyra [1] geometry.

In order to study the evolution of the universe, many authors constructed cosmological models containing a viscous fluid. The presence of viscosity in the fluid introduces many interesting features in the dynamics of homogeneous cosmological models. The possibility of bulk viscosity leading to inflationary like solutions in general relativistic FRW models has been discussed by several authors [13-17]. Roy and Tiwari [18], Mohanty and Pattanaik [19], Mohanty and Pradhan [20], Singh and Shriram [21], and Sing [22] are some of the authors who have investigated cosmological models with bulk viscosity in general relativity. Wang [23-25], Bali and Dave [26], Bali and Pradhan [27], Tripathy et al. [28], Tripathy et al. [29], and recently Rao et al. [30] have studied various Bianchi type cosmological models in the presence of cosmic strings and bulk viscosity.

Bianchi type spacetimes play a vital role in understanding and description of the early stages of evolution of the universe. In particular, the study of Bianchi types II, VIII, and IX universes is important because familiar solutions like FRW universe with positive curvature, the de Sitter universe, the Taub-Nut solutions, and so forth, correspond to Bianchi types II, VIII, and IX spacetimes. Bali and Dave [31], and Bali and Yadav [32] studied Bianchi type IX string as well as viscous fluid models in general relativity. Reddy et al. [33] studied Bianchi types II, VIII, and IX models in scale covariant theory of gravitation. Shanthi and Rao [34] studied Bianchi types VIII and IX models in Lyttleton-Bondi Universe. Also Rao and Sanyasi Raju [35], and Sanyasi Raju and Rao [36] have studied Bianchi types VIII, and IX models in Zero mass scalar fields and self-creation cosmology. Rahaman et al. [37] have investigated Bianchi type IX string cosmological model in a theory of gravitation formulated by Sen [3] based on Lyra [1] manifold. Rao et al. [38-40] have obtained Bianchi types II, VIII, and IX string, perfect fluid cosmological models in Saez-Ballester theory of gravitation, and string cosmological models in general relativity as well as self-creation theory of gravitation, respectively.

In this paper, we will discuss Bianchi types II, VIII, and IX string cosmological models with bulk viscosity in a theory of gravitation proposed by Sen [3] based on Lyra [1] geometry.

\section{Metric and Energy Momentum Tensor}

We consider a spatially homogeneous Bianchi types II, VIII, and IX metrics of the following form:

$$
d s^{2}=-d t^{2}+R^{2}\left[d \theta^{2}+f^{2}(\theta) d \phi^{2}\right]+S^{2}[d \varphi+h(\theta) d \phi]^{2},
$$

where $(\theta, \phi, \varphi)$ are the Eulerian angles, and $R$ and $S$ are functions of $t$ only. 
It represents, Bianchi type II if $f(\theta)=1$ and $h(\theta)=\theta$, Bianchi type VIII if $f(\theta)=\cosh \theta$ and $h(\theta)=\sinh \theta$, and Bianchi type IX if $f(\theta)=\sin \theta$ and $h(\theta)=\cos \theta$.

The energy momentum tensor for a bulk viscous fluid containing one-dimensional string as

$$
\begin{gathered}
T_{i j}=(\rho+\bar{p}) u_{i} u_{j}+\bar{p} g_{i j}-\lambda x_{i} x_{j} \\
\bar{p}=p-3 \xi H
\end{gathered}
$$

is the total pressure which includes the proper pressure, $\rho$ is the rest energy density of the system, $\lambda$ is tension in the string, $\xi(t)$ is the coefficient of bulk viscosity, $3 \xi H$ is usually known as bulk viscous pressure, $H$ is the Hubble parameter, $\beta$ the gauge function, $u^{i}=\delta_{4}^{i}$ is the four velocity vector and $x^{i}$ is a space-like vector which represents the anisotropic directions of the string.

Here $u^{i}$ and $x^{i}$ satisfy the

$$
\begin{gathered}
g_{i j} u^{i} u^{j}=-1, \\
g_{i j} x^{i} x^{j}=1, \\
u^{i} x_{i}=0 .
\end{gathered}
$$

We assume the string to be lying along the $z$-axis. The one-dimensional strings are assumed to be loaded with particles and the particle energy density is $\rho_{p}=\rho-\lambda$.

In a commoving coordinate system, we get

$$
T_{1}^{1}=T_{2}^{2}=\bar{p}, \quad T_{3}^{3}=\bar{p}-\lambda, \quad T_{4}^{4}=-\rho,
$$

where $\rho, \lambda, \bar{p}$, and $\phi$ are functions of time " $t$ " only.

\section{Solutions of Field Equations}

Now with the help of (2.2) to (2.7), the field (1.1) for the metric (2.1) can be written as

$$
\begin{gathered}
\frac{\ddot{R}}{R}+\frac{\ddot{S}}{S}+\frac{\dot{R} \dot{S}}{R S}+\frac{S^{2}}{4 R^{4}}+\frac{3}{4} \beta^{2}=\bar{p} \\
2 \frac{\ddot{R}}{R}+\frac{\dot{R}^{2}+\delta}{R^{2}}-\frac{3 S^{2}}{4 R^{4}}+\frac{3}{4} \beta^{2}=\bar{p}-\lambda, \\
2 \frac{\dot{R} \dot{S}}{R S}+\frac{\dot{R}^{2}+\delta}{R^{2}}-\frac{S^{2}}{4 R^{4}}-\frac{3}{4} \beta^{2}=-\rho .
\end{gathered}
$$

Here the over head dot denotes differentiation with respect to " $t$ ".

When $\delta=0,-1,+1$, the fields, (3.1) to (3.3) correspond to the Bianchi types II, VIII, and IX universes, respectively. 
By taking the transformation $d t=R^{2} S d T$, the previous fields (3.1) to (3.3) can be written as

$$
\begin{gathered}
\left(\frac{R^{\prime}}{R}\right)^{\prime}+\left(\frac{S^{\prime}}{S}\right)^{\prime}-\left(\frac{R^{\prime}}{R}\right)^{2}-2\left(\frac{R^{\prime} S^{\prime}}{R S}\right)+\frac{S^{4}}{4}+\frac{3}{4} \beta^{2} R^{4} S^{2}=\bar{p} R^{4} S^{2}, \\
2\left(\frac{R^{\prime}}{R}\right)^{\prime}-\left(\frac{R^{\prime}}{R}\right)^{2}-2\left(\frac{R^{\prime} S^{\prime}}{R S}\right)-\frac{3 S^{4}}{4}+\delta R^{2} S^{2}+\frac{3}{4} \beta^{2} R^{4} S^{2}=(\bar{p}-\lambda) R^{4} S^{2}, \\
\left(\frac{R^{\prime}}{R}\right)^{2}+2\left(\frac{R^{\prime} S^{\prime}}{R S}\right)-\frac{S^{4}}{4}+\delta R^{2} S^{2}-\frac{3}{4} \beta^{2} R^{4} S^{2}=-\rho R^{4} S^{2} .
\end{gathered}
$$

Here the over head dash denotes differentiation with respect to " $T$ ".

The fields (3.4) to (3.6) are only three independent equations with seven unknowns $R, S, \rho, \lambda, \bar{p}, \beta$, and $\xi$, which are functions of " $T$ ". Since these equations are highly nonlinear in nature, in order to get a deterministic solution, we take the following plausible physical conditions.

(1) The shear scalar $\sigma$ is proportional to scalar expansion $\theta$, so that we can take a linear relationship between the metric potentials $R$ and $S$, that is,

$$
R=S^{n}
$$

where $n$ is an arbitrary constant.

(2) A more general relationship between the proper rest energy density $\rho$ and string tension density $\lambda$ is taken to be

$$
\rho=r \lambda
$$

where $r$ is an arbitrary constant which can take both positive and negative values. The negative value of $r$ leads to the absence of strings in the universe and the positive value shows the presence of one dimensional string in the cosmic fluid. The energy density of the particles attached to the strings is

$$
\rho_{p}=\rho-\lambda=(r-1) \lambda
$$

(3) For a barotropic fluid, the combined effect of the proper pressure and the barotropic bulk viscous pressure can be expressed as

$$
\bar{p}=p-3 \xi H=(\in \rho),
$$

where $\epsilon=\epsilon_{0}-\varsigma$ and $p=\epsilon_{0} \rho\left(0 \leq \epsilon_{0} \leq 1\right)$. 
Using (3.7), the fields (3.4), (3.5), and (3.6) can be written as

$$
\begin{gathered}
\frac{S^{\prime \prime}}{S}(n+1)-\frac{S^{\prime 2}}{S^{2}}\left(n^{2}+3 n+1\right)+\frac{S^{4}}{4}+\frac{3}{4} \beta^{2} S^{4 n+2}=\bar{p} S^{4 n+2}, \\
\frac{S^{\prime \prime}}{S}(2 n)-\frac{S^{\prime 2}}{S^{2}}\left(n^{2}+4 n\right)-\frac{3 S^{4}}{4}+\delta S^{2 n+2}+\frac{3}{4} \beta^{2} S^{4 n+2}=(\bar{p}-\lambda) S^{4 n+2}, \\
\frac{S^{\prime 2}}{S^{2}}\left(n^{2}+2 n\right)-\frac{S^{4}}{4}+\delta S^{2 n+2}-\frac{3}{4} \beta^{2} S^{4 n+2}=-\rho S^{4 n+2}
\end{gathered}
$$

From (3.11) and (3.12), we get

$$
\frac{S^{\prime \prime}}{S}(1-n)-\frac{S^{2}}{S^{2}}(1-n)+S^{4}-\delta S^{2 n+2}=\lambda S^{4 n+2}
$$

From (3.11), (3.13), (3.8), and (3.10), we obtain

$$
\left(\frac{S^{\prime}}{S}\right)^{\prime}(1+n)+\delta S^{2 n+2}=r \lambda(\in-1) S^{4 n+2} .
$$

From (3.14) and (3.15), we get

$$
C_{1}\left(\frac{S^{\prime}}{S}\right)^{\prime}+C_{2} S^{4}-C_{3} \delta S^{2 n+2}=0
$$

where $C_{1}=r(\in-1)(1-n)-(1+n), C_{2}=r(\in-1), C_{3}=r(\in-1)+1$.

\subsection{Bianchi Type II $(\delta=0)$ Cosmological Model}

If $\delta=0$, (3.16) can be written as

$$
C_{1}\left(\frac{S^{\prime}}{S}\right)^{\prime}+C_{2} S^{4}=0
$$

where $C_{1}=r(\in-1)(1-n)-(1+n), C_{2}=r(\in-1)$.

From (3.17), with suitable substitution, we get

$$
S^{\prime 2}=W^{2} S^{6}+r^{2} S^{2}
$$

where $\gamma^{2}$ is an integrating constant and

$$
W^{2}=\frac{-C_{2}}{2 C_{1}}=\frac{-r(\in-1)}{2(r(\epsilon-1)(1-n)-(1+n))} .
$$


From (3.18), we get

$$
S^{2}=\left(\frac{C_{3}}{2 W}\right)\left(\operatorname{coth}^{2}\left(C_{3} T\right)-1\right)^{1 / 2}
$$

where $C_{3}=-2 \gamma$.

From (3.20) and (3.7), we get

$$
R^{2}=\left(\frac{C_{3}}{2 W}\right)^{n}\left(\operatorname{coth}^{2}\left(C_{3} T\right)-1\right)^{n / 2}
$$

From (3.11), (3.13), and (3.10), we obtain

$$
\left(\frac{S^{\prime}}{S}\right)^{\prime}(1+n)=\rho(\in-1) S^{4 n+2}
$$

From (3.22), we get the energy density:

$$
\rho=\frac{(1+n)(2 W)^{1+2 n}}{2(\in-1)\left(C_{3}\right)^{2 n-1}}\left(\operatorname{coth}^{2}\left(C_{3} T\right)-1\right)^{(1-2 n) / 2} .
$$

The total pressure is given by

$$
\bar{p}=\in \rho=\frac{\in(1+n)(2 W)^{1+2 n}}{2(\in-1)\left(C_{3}\right)^{2 n-1}}\left(\operatorname{coth}^{2}\left(C_{3} T\right)-1\right)^{(1-2 n) / 2} .
$$

The proper pressure is given by

$$
p=\epsilon_{0} \rho=\frac{\epsilon_{0}(1+n)(2 W)^{1+2 n}}{2(\in-1)\left(C_{3}\right)^{2 n-1}}\left(\operatorname{coth}^{2}\left(C_{3} T\right)-1\right)^{(1-2 n) / 2} .
$$

From (3.11), (3.13), and (3.10), we obtain

$$
\frac{S^{\prime 2}}{S^{2}}\left(2 n^{2}+4 n\right)-\left(\frac{S^{\prime}}{S}\right)^{\prime}(1+n)-\frac{2 S^{4}}{4}-\frac{6}{4} \beta^{2} S^{4 n+2}=-\rho(1+\epsilon) S^{4 n+2}
$$

From (3.26), we get the displacement vector:

$$
\beta^{2}=\left[\begin{array}{c}
\frac{C_{3}^{1-2 n}}{3(2 W)^{1-2 n}}\left[\left(4 n^{2} W^{2}-1\right)+8 W^{2}\left(\frac{n \in+1}{\in-1}\right)\right]\left(\operatorname{coth}^{2}\left(C_{3} T\right)-1\right)^{(1-2 n) / 2} \\
+\frac{(2 W)^{1+2 n}}{3 C_{3}^{2 n-1}}\left(n^{2}+2 n\right)\left(\operatorname{coth}^{2}\left(C_{3} T\right)-1\right)^{-(2 n+1) / 2}
\end{array}\right] .
$$


From (3.12), (3.20), (3.24), and (3.27), we get the string tension density:

$$
\lambda=\frac{C_{3}^{1-2 n}}{(2 W)^{1-2 n}}\left[2 W^{2}(1-n)+1\right]\left(\operatorname{coth}^{2}\left(C_{3} T\right)-1\right)^{(1-2 n) / 2} .
$$

The particle energy density is given by

$$
\rho_{p}=\rho-\lambda=(r-1) \lambda=\frac{(r-1) C_{3}{ }^{1-2 n}}{(2 W)^{1-2 n}}\left(2 W^{2}(1-n)+1\right)\left(\operatorname{coth}^{2}\left(C_{3} T\right)-1\right)^{(1-2 n) / 2} .
$$

The coefficient of bulk viscosity is given by

$$
\xi=\frac{\zeta(1+n)(2 w)^{1+2 n}}{(\epsilon-1)(2 n+1) C_{3}^{2 n}}\left(\operatorname{coth}^{2}\left(C_{3} t\right)-1\right)^{(1-2 n) / 2} \operatorname{coth}^{-1}\left(C_{3} t\right) .
$$

The components of Hubble parameter $H_{1}, H_{2}$, and $H_{3}$ are given by

$$
H_{1}=\frac{R^{\prime}}{R}=\frac{n C_{3}}{2} \operatorname{coth}\left(C_{3} T\right), \quad H_{2}=\frac{S^{\prime}}{S}=\frac{C_{3}}{2} \operatorname{coth}\left(C_{3} T\right), \quad H_{3}=\frac{R^{\prime}}{R}=\frac{n C_{3}}{2} \operatorname{coth}\left(C_{3} T\right) .
$$

Therefore the generalized mean Hubble parameter $(H)$ is

$$
H=\frac{1}{3}\left(H_{1}+H_{2}+H_{3}\right)=\frac{(2 n+1) C_{3}}{6} \operatorname{coth}\left(C_{3} T\right) .
$$

The metric (2.1), in this case, can be written as

$$
\begin{aligned}
d s^{2}= & -\left(\left(\frac{C_{3}}{2 W}\right)^{(2 n+1)}\left(\operatorname{coth}^{2}\left(C_{3} T\right)-1\right)^{(2 n+1) / 2}\right) d T^{2}+\left(\left(\frac{C_{3}}{2 W}\right)^{n}\left(\operatorname{coth}^{2}\left(C_{3} T\right)-1\right)^{n / 2}\right) \\
& \times\left(d \theta^{2}+d \phi^{2}\right)+\left(\left(\frac{C_{3}}{2 W}\right)\left(\operatorname{coth}^{2}\left(C_{3} T\right)-1\right)^{1 / 2}\right)(d \varphi+\theta d \phi)^{2} .
\end{aligned}
$$

Thus (3.33) together with (3.23), (3.24), and (3.28) constitutes a Bianchi type-II string cosmological model with bulk viscosity in Sen [3] theory of gravitation.

\subsection{Bianchi Type VIII $(\delta=-1)$ Cosmological Model}

If $\delta=-1$, (3.16) can be written as

$$
C_{1}\left(\frac{S^{\prime}}{S}\right)^{\prime}+C_{2} S^{4}+C_{3} S^{2 n+2}=0
$$

where $C_{1}=r(\in-1)(1-n)-(1+n), C_{2}=r(\in-1), C_{3}=r(\in-1)+1$. 
From (3.34), with suitable substitution and for $n=1$, we get

$$
S^{\prime 2}=W^{2} S^{6}+r^{2} S^{2},
$$

where $\gamma^{2}$ is an integrating constant and

$$
W^{2}=\frac{-\left(C_{2}+C_{3}\right)}{2 C_{1}}=\frac{(2 r(\in-1)+1)}{4} .
$$

From (3.35), we get

$$
S^{2}=R^{2}=\left(\frac{C_{4}}{2 W}\right)\left(\operatorname{coth}^{2}\left(C_{4} T\right)-1\right)^{1 / 2}, \quad \text { where } C_{4}=-2 \gamma
$$

From (3.11), (3.13), and (3.10), we obtain

$$
2\left(\frac{S^{\prime}}{S}\right)^{\prime}-S^{4}=\rho(\in-1) S^{6}
$$

From (3.38), we get the energy density:

$$
\rho=\frac{2 W}{C_{4}(\in-1)}\left(4 W^{2}-1\right)\left(\operatorname{coth}^{2}\left(C_{4} T\right)-1\right)^{-1 / 2} .
$$

The total pressure is given by

$$
\bar{p}=\in \rho=\frac{2 \in W}{C_{4}(\in-1)}\left(4 W^{2}-1\right)\left(\operatorname{coth}^{2}\left(C_{4} T\right)-1\right)^{-1 / 2} .
$$

The proper pressure is given by

$$
p=\epsilon_{0} \rho=\frac{2 \epsilon_{0} W}{C_{4}(\in-1)}\left(4 W^{2}-1\right)\left(\operatorname{coth}^{2}\left(C_{4} T\right)-1\right)^{-1 / 2} .
$$

From (3.11), (3.13), and (3.10), we obtain

$$
6 \frac{S^{\prime 2}}{S^{2}}-2\left(\frac{S^{\prime}}{S}\right)^{\prime}-\frac{6 S^{4}}{4}-\frac{6}{4} \beta^{2} S^{6}=-\rho(1+\epsilon) S^{6} .
$$

From (3.42), we get the displacement vector

$$
\beta^{2}=\left[\frac{2 W}{3 C_{4}}\left[\left(4 W^{2}-3\right)+2\left(\frac{\epsilon+1}{\epsilon-1}\right)\left(4 W^{2}-1\right)\right]\left(\operatorname{coth}^{2}\left(C_{4} T\right)-1\right)^{-1 / 2}+\frac{8 W^{3}}{C_{4}}\left(\operatorname{coth}^{2}\left(C_{4} T\right)-1\right)^{-3 / 2}\right] \text {. }
$$


From (3.12), (3.37), (3.40), and (3.43), we get the string tension density:

$$
\lambda=\frac{4 W}{C_{4}}\left(\operatorname{coth}^{2}\left(C_{4} T\right)-1\right)^{-1 / 2}
$$

The particle energy density is given by

$$
\rho_{p}=\rho-\lambda=(r-1) \lambda=\frac{(r-1) 4 W}{C_{4}}\left(\operatorname{coth}^{2}\left(C_{4} T\right)-1\right)^{-1 / 2}
$$

The coefficient of bulk viscosity is given by

$$
\xi=\frac{4 \zeta W}{3 C_{4}^{2}(\in-1)}\left(4 W^{2}-1\right)\left(\operatorname{coth}^{2}\left(C_{4} T\right)-1\right)^{-1 / 2} \operatorname{coth}^{-1}\left(C_{4} T\right)
$$

The components of Hubble parameter $H_{1}, H_{2}$, and $H_{3}$ are given by

$$
H_{1}=H_{2}=H_{3}=\frac{R^{\prime}}{R}=\frac{S^{\prime}}{S}=\frac{C_{4}}{2} \operatorname{coth}\left(C_{4} T\right)
$$

Therefore the generalized mean Hubble parameter $(H)$ is

$$
H=\frac{1}{3}\left(H_{1}+H_{2}+H_{3}\right)=\frac{\mathrm{c}_{4}}{2} \operatorname{coth}\left(C_{4} T\right)
$$

The metric (2.1), in this case, can be written as

$$
\begin{aligned}
d s^{2}= & -\left(\left(\frac{C_{4}}{2 W}\right)^{3}\left(\operatorname{coth}^{2}\left(C_{4} T\right)-1\right)^{3 / 2}\right) d T^{2}+\left(\left(\frac{C_{4}}{2 W}\right)\left(\operatorname{coth}^{2}\left(C_{4} T\right)-1\right)^{1 / 2}\right) \\
& \times\left(d \theta^{2}+\cosh ^{2} \theta d \phi^{2}\right)+\left(\left(\frac{C_{4}}{2 W}\right)\left(\operatorname{coth}^{2}\left(C_{4} T\right)-1\right)^{1 / 2}\right)(d \varphi+\sinh \theta d \phi)^{2}
\end{aligned}
$$

Thus (3.49) together with (3.39), (3.40), and (3.44) constitutes a Bianchi type VIII string cosmological model with bulk viscosity in Sen [3] theory of gravitation.

\subsection{Bianchi Type IX $(\delta=1)$ Cosmological Model}

If $\delta=1$, (3.16) can be written as

$$
C_{1}\left(\frac{S^{\prime}}{S}\right)^{\prime}+C_{2} S^{4}-C_{3} S^{2 n+2}=0
$$


where

$$
\begin{gathered}
C_{1}=r(\in-1)(1-n)-(1+n), \\
C_{2}=r(\in-1), \\
C_{3}=r(\in-1)+1 .
\end{gathered}
$$

From (3.50), with suitable substitution and for $n=1$, we get

$$
S^{\prime 2}=W^{2} S^{6}+r^{2} S^{2},
$$

where $\gamma^{2}$ is an integrating constant and

$$
W^{2}=\frac{\left(C_{3}-C_{2}\right)}{2 C_{1}}=\frac{-1}{4} .
$$

Integrating (3.54), we get

$$
S^{2}=R^{2}=\left(\frac{C_{4}}{2 W}\right)\left(\operatorname{coth}^{2}\left(C_{4} T\right)-1\right)^{1 / 2},
$$

where

$$
C_{4}=-2 \gamma \text {. }
$$

From (3.11), (3.13), and (3.10), we obtain

$$
2\left(\frac{S^{\prime}}{S}\right)^{\prime}+S^{4}=\rho(\in-1) S^{6} .
$$

From (3.58), we get the energy density:

$$
\rho=\frac{2 W}{C_{4}(\in-1)}\left(4 W^{2}+1\right)\left(\operatorname{coth}^{2}\left(C_{4} T\right)-1\right)^{-1 / 2} .
$$

The total pressure is given by

$$
\bar{p}=\in \rho=\frac{2 \in W}{C_{4}(\in-1)}\left(4 W^{2}+1\right)\left(\operatorname{coth}^{2}\left(C_{4} T\right)-1\right)^{-1 / 2} .
$$

The proper pressure is given by

$$
p=\epsilon_{0} \rho=\frac{2 \epsilon_{0} W}{C_{4}(\in-1)}\left(4 W^{2}+1\right)\left(\operatorname{coth}^{2}\left(C_{4} T\right)-1\right)^{-1 / 2} .
$$

Since $W^{2}=\left(C_{3}-C_{2}\right) / 2 C_{1}=(-1 / 4)$, from (3.59) to (3.61) and (3.12), we can observe that the energy density $\rho$, total pressure $\bar{p}$, proper pressure $p$, and string tension density $\lambda$ will vanish. 
From (3.11), (3.13), and (3.10), we obtain

$$
6 \frac{S^{\prime 2}}{S^{2}}-2\left(\frac{S^{\prime}}{S}\right)^{\prime}+\frac{2 S^{4}}{4}-\frac{6}{4} \beta^{2} S^{6}=-\rho(1+\epsilon) S^{6}
$$

From (3.62), we get the displacement vector:

$$
\beta^{2}=\frac{-2 W}{C_{4}}\left(\operatorname{coth}^{2}\left(C_{4} T\right)-1\right)^{-3 / 2}
$$

The coefficient of bulk viscosity is given by

$$
\xi=\frac{4 \zeta W}{3 C_{4}^{2}(\in-1)}\left(4 W^{2}+1\right)\left(\operatorname{coth}^{2}\left(C_{4} T\right)-1\right)^{-1 / 2} \operatorname{coth}^{-1}\left(C_{4} T\right)
$$

Since $W^{2}=\left(C_{3}-C_{2}\right) / 2 C_{1}=(-1 / 4)$, the coefficient of bulk viscosity $\xi$ will vanish.

The components of Hubble parameter $H_{1}, H_{2}$, and $H_{3}$ are given by

$$
H_{1}=H_{2}=H_{3}=\frac{R^{\prime}}{R}=\frac{S^{\prime}}{S}=\frac{C_{4}}{2} \operatorname{coth}\left(C_{4} T\right)
$$

Therefore the generalized mean Hubble parameter $(H)$ is

$$
H=\frac{1}{3}\left(H_{1}+H_{2}+H_{3}\right)=\frac{C_{4}}{2} \operatorname{coth}\left(C_{4} T\right)
$$

The metric (2.1), in this case, can be written as

$$
\begin{aligned}
d s^{2}= & -\left(\left(\frac{C_{4}}{2 W}\right)^{3}\left(\operatorname{coth}^{2}\left(C_{4} T\right)-1\right)^{3 / 2}\right) d T^{2}+\left(\left(\frac{C_{4}}{2 W}\right)\left(\operatorname{coth}^{2}\left(C_{4} T\right)-1\right)^{1 / 2}\right) \\
& \times\left(d \theta^{2}+\sin ^{2} \theta d \phi^{2}\right)+\left(\left(\frac{C_{4}}{2 W}\right)\left(\operatorname{coth}^{2}\left(C_{4} T\right)-1\right)^{1 / 2}\right)(d \varphi+\cos \theta d \phi)^{2}
\end{aligned}
$$

Thus (3.67) together with (3.59) and (3.60) constitutes a Bianchi type IX vacuum cosmological model in Sen [3] theory of gravitation.

\subsection{The Cosmological Models in the Absence of Bulk Viscosity}

It is interesting to note that in the absence of bulk viscosity, by taking $\zeta=0$ in (3.30), (3.46), and (3.64), we get the Bianchi types II, VIII, and IX perfect fluid string cosmological models, respectively, and if we assign the value zero to $\in$ and $\epsilon_{0}$, the present models reduce to string cosmological models in Sen [3] theory of gravitation. 


\section{Physical and Geometrical Properties}

\subsection{Bianchi Type II Cosmological Model $(\delta=0)$}

The spatial volume for the model is

$$
V=(-g)^{1 / 2}\left(\left(\frac{C_{3}}{2 W}\right)^{2 n+1 / 2}\left(\operatorname{coth}^{2}\left(C_{3} T\right)-1\right)^{2 n+1 / 4}\right) .
$$

The expression for expansion scalar $\theta$ calculated for the flow vector $u^{i}$ is given by

$$
\theta=u_{; i}^{i}=\frac{(2 n+1)(2 W)^{2 n+1 / 2}}{2\left(C_{3}\right)^{2 n-1 / 2}}\left(\operatorname{coth}^{2}\left(C_{3} T\right)-1\right)^{-(2 n+1) / 4} \operatorname{coth}\left(C_{3} T\right),
$$

and the shear $\sigma$ is given by

$$
\sigma^{2}=\frac{1}{2} \sigma^{i j} \sigma_{i j}=\frac{5(2 n+1)^{2}(2 W)^{2 n+1}}{72 C_{3}^{2 n-1}}\left(\operatorname{coth}^{2}\left(C_{3} T\right)-1\right)^{-(2 n+1) / 2} \operatorname{coth}^{2}\left(C_{3} T\right) .
$$

The deceleration parameter $q$ is given by

$$
\begin{aligned}
q= & \left(-3 \theta^{-2}\right)\left(\theta_{, i} u^{i}+\frac{1}{3} \theta^{2}\right) \\
= & \left(\frac{6}{2 n+1}\right)\left(\frac{C_{3}}{2 W}\right)^{2 n+1 / 2}\left(\operatorname{coth}^{2}\left(C_{3} T\right)-1\right)^{(2 n+1) / 4} \operatorname{coth}^{-2}\left(C_{3} T\right) \\
& \times\left(\frac{2 n+1}{2} \operatorname{coth}^{2}\left(C_{3} T\right)+\cos \operatorname{ech}^{2}\left(C_{3} T\right)\right)-1
\end{aligned}
$$

\subsection{Bianchi Type VII Cosmological Model $(\delta=-1)$}

The spatial volume for the model is

$$
V=(-g)^{1 / 2}=\left(\left(\frac{C_{4}}{2 W}\right)^{3 / 2}\left(\operatorname{coth}^{2}\left(C_{4} T\right)-1\right)^{3 / 4}\right) \cosh \theta
$$

The expression for expansion scalar $\theta$ calculated for the flow vector $u^{i}$ is given by

$$
\theta=u_{; i}^{i}=\frac{3(2 W)^{3 / 2}}{2 C_{4}{ }^{1 / 2}}\left(\operatorname{coth}^{2}\left(C_{4} T\right)-1\right)^{-3 / 4} \operatorname{coth}\left(C_{4} T\right),
$$

and the shear $\sigma$ is given by

$$
\sigma^{2}=\frac{1}{2} \sigma^{i j} \sigma_{i j}=\frac{5 W^{3}}{C_{4}}\left(\operatorname{coth}^{2}\left(C_{4} T\right)-1\right)^{-3 / 2} \operatorname{coth}^{2}\left(C_{4} T\right) .
$$


The deceleration parameter $q$ is given by

$$
\begin{aligned}
q & =\left(-3 \theta^{-2}\right)\left(\theta_{, i} u^{i}+\frac{1}{3} \theta^{2}\right) \\
& =2\left(\frac{C_{4}}{2 W}\right)^{3 / 2}\left(\operatorname{coth}^{2}\left(C_{4} T\right)-1\right)^{3 / 4} \operatorname{coth}^{-2}\left(C_{4} T\right)\left(\frac{3}{2} \operatorname{coth}^{2}\left(C_{4} T\right)+\cos \operatorname{ech}^{2}\left(C_{4} T\right)\right)-1 .
\end{aligned}
$$

\subsection{Bianchi Type IX Cosmological Model $(\delta=1)$}

The spatial volume for the model is

$$
V=(-g)^{1 / 2}=\left(\left(\frac{C_{4}}{2 W}\right)^{3 / 2}\left(\operatorname{coth}^{2}\left(C_{4} T\right)-1\right)^{3 / 4}\right) \sin \theta
$$

The expression for expansion scalar $\theta$ calculated for the flow vector $u^{i}$ is given by

$$
\theta=u_{; i}^{i}=\frac{3(2 W)^{3 / 2}}{2\left(C_{4}\right)^{1 / 2}}\left(\operatorname{coth}^{2}\left(C_{4} T\right)-1\right)^{-3 / 4} \operatorname{coth}\left(C_{4} T\right),
$$

and the shear $\sigma$ is given by

$$
\sigma^{2}=\frac{1}{2} \sigma^{i j} \sigma_{i j}=\frac{5 W^{3}}{C_{4}}\left(\operatorname{coth}^{2}\left(C_{4} T\right)-1\right)^{-3 / 2} \operatorname{coth}^{2}\left(C_{4} T\right) .
$$

The deceleration parameter $q$ is given by

$$
\begin{aligned}
q & =\left(-3 \theta^{-2}\right)\left(\theta_{i, i} u^{i}+\frac{1}{3} \theta^{2}\right) \\
& =2\left(\frac{C_{4}}{2 W}\right)^{3 / 2}\left(\operatorname{coth}^{2}\left(C_{4} T\right)-1\right)^{3 / 4} \operatorname{coth}^{-2}\left(C_{4} T\right)\left(\frac{3}{2} \operatorname{coth}^{2}\left(C_{4} T\right)+\operatorname{cosech}^{2}\left(C_{4} T\right)\right)-1 .
\end{aligned}
$$

\section{Conclusion}

In this paper we have presented Bianchi types II, VIII, and IX string cosmological models with bulk viscosity in a theory of gravitation proposed by Sen [3] based on Lyra [1] geometry. It is observed that in case of Bianchi type IX only vacuum cosmological model exists. The models have no initial singularity at $T=0$ and the spatial volume is decreasing as time $T$ increases; that is, all the three models are contracting. For Bianchi type II cosmological model, the energy density $\rho$ and the total pressure $\bar{p}$ will tend to infinity as $T$ approaches to zero, if $2 n-1<0$. Also the expansion scalar $\theta$ and the shear scalar $\sigma$ for this model will tend to infinity as $T$ approaches to zero, if $2 n+1<0$. But the energy density $\rho$, the total pressure $\bar{p}$, the expansion scalar $\theta$, and the shear scalar $\sigma$ will tend to zero as $T$ approaches to zero for Bianchi types VIII 
and IX cosmological models. Since the deceleration parameter $q$ is greater than zero for all the models, they represent decelerating universes. Since $\lim _{T \rightarrow \infty} \sigma^{2} / \theta^{2}=5 / 18 \neq 0$, the models do not approach isotropy for large values of $T$.

\section{Acknowledgment}

K. V. S. Sireesha is grateful to the Department of Science and Technology (DST), New Delhi, India for providing INSPIRE fellowship.

\section{References}

[1] G. Lyra, "Über eine modifikation der riemannschen geometrie," Mathematische Zeitschrift, vol. 54, pp. 52-64, 1951.

[2] H. Weyl, "Gravitation und Elektrizität," Sitzungsberichte der Königlich Preussischen Akademie der Wissenschaften zu Berlin, vol. 465, 1918.

[3] D. K. Sen, "A static cosmological model," Zeitschrift für Physik C, vol. 149, pp. 311-323, 1957.

[4] D. K. Sen and K. A. Dunn, "A scalar-tensor theory of gravitation in a modified Riemannian manifold," Journal of Mathematical Physics, vol. 12, no. 4, pp. 578-586, 1971.

[5] W. D. Halford, "Scalar-tensor theory of gravitation in a Lyra manifold," Journal of Mathematical Physics, vol. 13, no. 11, pp. 1699-1703, 1972.

[6] P. S. Letelier, "String cosmologies," Physical Review D, vol. 28, no. 10, pp. 2414-2419, 1983.

[7] K. D. Krori, T. Chaudhury, C. R. Mahanta, and A. Mazumdar, "Some exact solutions in string cosmology," General Relativity and Gravitation, vol. 22, no. 2, pp. 123-130, 1990.

[8] P. Mahanta and A. Mukherjee, "String models in Lyra geometry," Indian Journal of Pure and Applied Mathematics, vol. 32, no. 2, pp. 199-204, 2001.

[9] R. Bhattacharjee and K. K. Baruah, "String cosmologies with a scalar field," Indian Journal of Pure and Applied Mathematics, vol. 32, no. 1, pp. 47-53, 2001.

[10] D. R. K. Reddy and M. V. S. Rao, "A xially symmetric cosmic strings and domain walls in Lyra geometry," Astrophysics and Space Science, vol. 302, no. 1-4, pp. 157-160, 2006.

[11] G. Mohanty and K. L. Mahanta, "Five-dimensional axially symmetric string cosmological model in Lyra manifold," Astrophysics and Space Science, vol. 312, no. 3-4, pp. 301-304, 2007.

[12] V. U. M. Rao and T. Vinutha, "Axially symmetric cosmological models in a scalar tensor theory based on Lyra manifold," Astrophysics and Space Science, vol. 319, no. 2-4, pp. 161-167, 2009.

[13] J. D. Barrow, "The deflationary universe: an instability of the de Sitter universe," Physics Letters B, vol. 180, no. 4, pp. 335-339, 1986.

[14] T. Padmanabhan and S. M. Chitre, "Viscous universes," Physics Letters A, vol. 120, no. 9, pp. 433-436, 1987.

[15] D. Pavón, J. Bafaluy, and D. Jou, "Causal Friedmann-Robertson-Walker cosmology," Classical and Quantum Gravity, vol. 8, no. 2, pp. 347-360, 1991.

[16] R. Martens, “Dissipative cosmology," Classical and Quantum Gravity, vol. 12, no. 6, article 1455, 1995.

[17] J. A. S Lima, A. S. M Germano, and L. R. W Abrama, "FRW-type cosmologies with adiabatic matter creation," Physical Review D, vol. 53, pp. 4287-4297, 1996.

[18] S. R. Roy and O. P. Tiwari, "Some inhomogeneous viscous fluid cosmological models of plane symmetry," Indian Journal of Pure and Applied Mathematics, vol. 14, no. 2, pp. 233-243, 1983.

[19] G. Mohanty and R. R. Pattanaik, "Anisotropic, spatially homogeneous, bulk viscous cosmological model," International Journal of Theoretical Physics, vol. 30, no. 2, pp. 239-244, 1991.

[20] G. Mohanty and B. D. Pradhan, "Cosmological mesonic viscous fluid model," International Journal of Theoretical Physics, vol. 31, no. 1, pp. 151-160, 1992.

[21] J. K. Singh and R. Shri, "Plane-symmetric mesonic viscous fluid cosmological model," Astrophysics and Space Science, vol. 236, no. 2, pp. 277-284, 1996.

[22] J. Sing, “Some viscous fluid cosmological models," Il Nuovo Cimento B, vol. 120, no. 12, pp. 1251-1259, 2005.

[23] X.-X. Wang, "Locally rotationally symmetric bianchi type-I string cosmological model with bulk viscosity," Chinese Physics Letters, vol. 21, no. 7, pp. 1205-1207, 2004.

[24] X.-X. Wang, "Bianchi type-III string cosmological model with bulk viscosity in general relativity," Chinese Physics Letters, vol. 22, no. 1, pp. 29-32, 2005. 
[25] X.-X. Wang, "Bianchi type-III string cosmological model with bulk viscosity and magnetic field," Chinese Physics Letters, vol. 23, no. 7, pp. 1702-1704, 2006.

[26] R. Bali and S. Dave, "Bianchi type-III string cosmological model with bulk viscous fluid in general relativity," Astrophysics and Space Science, vol. 282, no. 2, pp. 461-467, 2002.

[27] R. Bali and A. Pradhan, "Bianchi type-III string cosmological models with time dependent bulk viscosity," Chinese Physics Letters, vol. 24, no. 2, article 079, pp. 585-588, 2007.

[28] S. K. Tripathy, S. K. Nayak, S. K. Sahu, and T. R. Routray, "Bulk viscous string cosmological models with electromagnetic field," Astrophysics and Space Science, vol. 321, no. 3-4, pp. 247-252, 2009.

[29] S. K. Tripathy, D. Behera, and T. R. Routray, "Anisotropic universe with cosmic strings and bulk viscosity," Astrophysics and Space Science, vol. 325, no. 1, pp. 93-97, 2010.

[30] V. U. M. Rao, G. Sree Devi Kumari, and K. V. S. Sireesha, "Anisotropic universe with cosmic strings and bulk viscosity in a scalar-tensor theory of gravitation," Astrophysics and Space Science, vol. 335, no. 2, pp. 635-638, 2011.

[31] R. Bali and S. Dave, “Bianchi type IX string cosmological model in general relativity,” Pramana: Journal of Physics, vol. 56, no. 4, pp. 513-518, 2001.

[32] R. Bali and M. K. Yadav, "Bianchi type-IX viscous fluid cosmological model in general relativity," Pramana: Journal of Physics, vol. 64, no. 2, pp. 187-196, 2005.

[33] D. R. K. Reddy, B. M. Patrudu, and R. Venkateswarlu, "Exact Bianchi type II, VIII and IX cosmological models in scale-covariant theory of gravitation," Astrophysics and Space Science, vol. 204, no. 1, pp. 155160, 1993.

[34] K. Shanthi and V. U. M. Rao, "Bianchi type-II and III models in self-creation cosmology," Astrophysics and Space Science, vol. 179, no. 1, pp. 147-153, 1991.

[35] V. U. M. Rao and Y. V. S. S. Sanyasi Raju, "Exact Bianchi-type VIII and IX models in the presence of zero-mass scalar fields," Astrophysics and Space Science, vol. 187, no. 1, pp. 113-117, 1992.

[36] Y. V. S. S. Sanyasi Raju and V. U. M. Rao, "Exact Bianchi-type VIII and IX models in the presence of the self-creation theory of cosmology," Astrophysics and Space Science, vol. 189, no. 1, pp. 39-43, 1992.

[37] F. Rahaman, S. Chakraborty, N. Begum, M. Hossain, and M. Kalam, "Bianchi-IX string cosmological model in Lyra geometry," Pramana: Journal of Physics, vol. 60, no. 6, pp. 1153-1159, 2003.

[38] V. U. M. Rao, M. Vijaya Santhi, and T. Vinutha, "Exact Bianchi type II, VIII and IX string cosmological models in Saez-Ballester theory of gravitation," Astrophysics and Space Science, vol. 314, no. 1-3, pp. 73-77, 2008.

[39] V. U. M. Rao, M. Vijaya Santhi, and T. Vinutha, “Exact Bianchi type-II, VIII and IX perfect fluid cosmological models in Saez-Ballester theory of gravitation," Astrophysics and Space Science, vol. 317, no. 1-2, pp. 27-30, 2008.

[40] V. U. M. Rao, M. Vijaya Santhi, and T. Vinutha, "Exact Bianchi type II, VIII and IX string cosmological models in General Relativity and self-creation theory of gravitation," Astrophysics and Space Science, vol. 317, no. 1-2, pp. 83-88, 2008. 


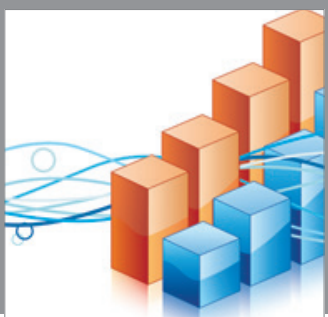

Advances in

Operations Research

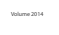

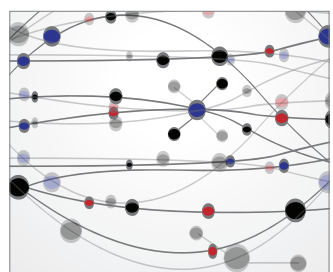

\section{The Scientific} World Journal
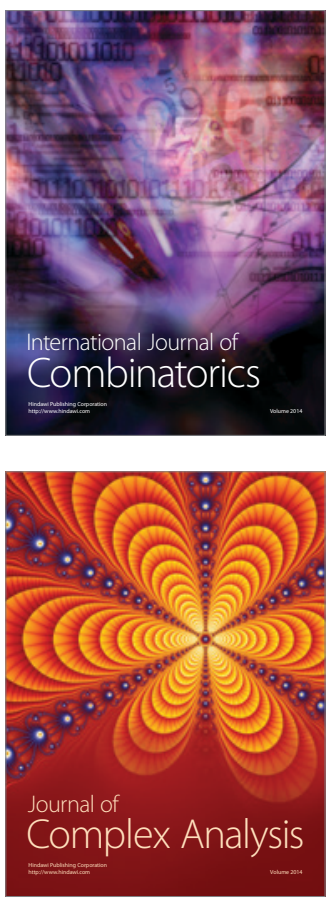

International Journal of

Mathematics and

Mathematical

Sciences
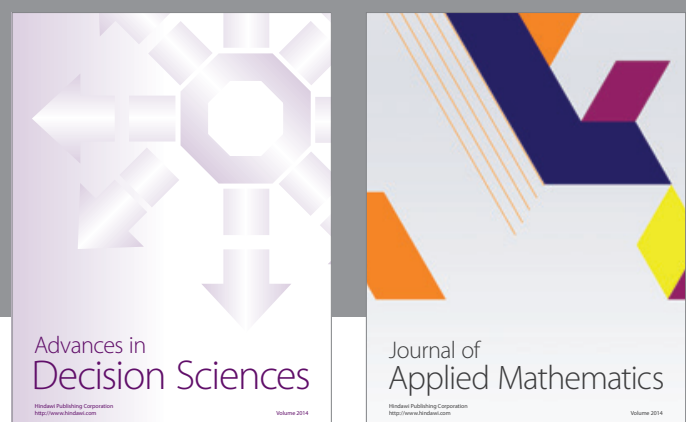

Journal of

Applied Mathematics
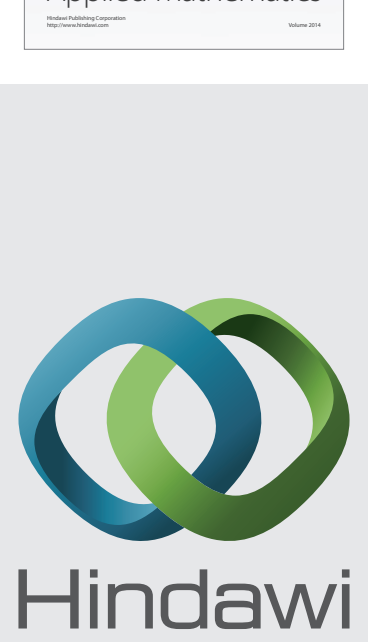

Submit your manuscripts at http://www.hindawi.com
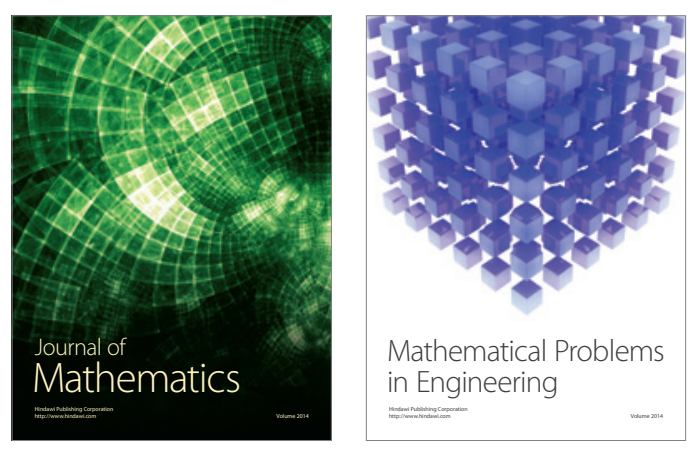

Mathematical Problems in Engineering
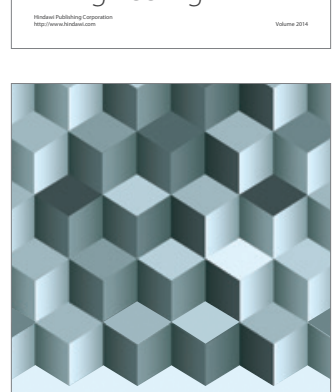

Journal of

Function Spaces
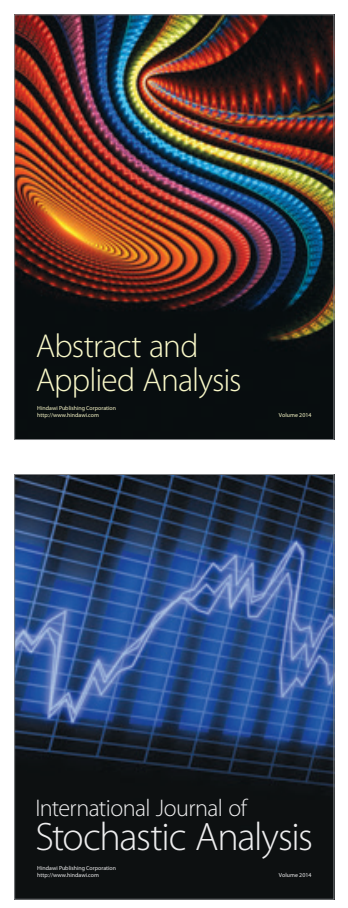

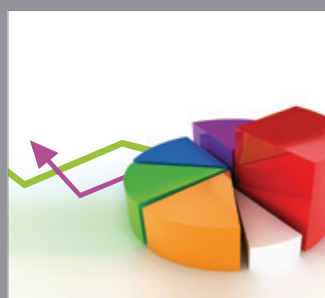

ournal of

Probability and Statistics

Promensencen
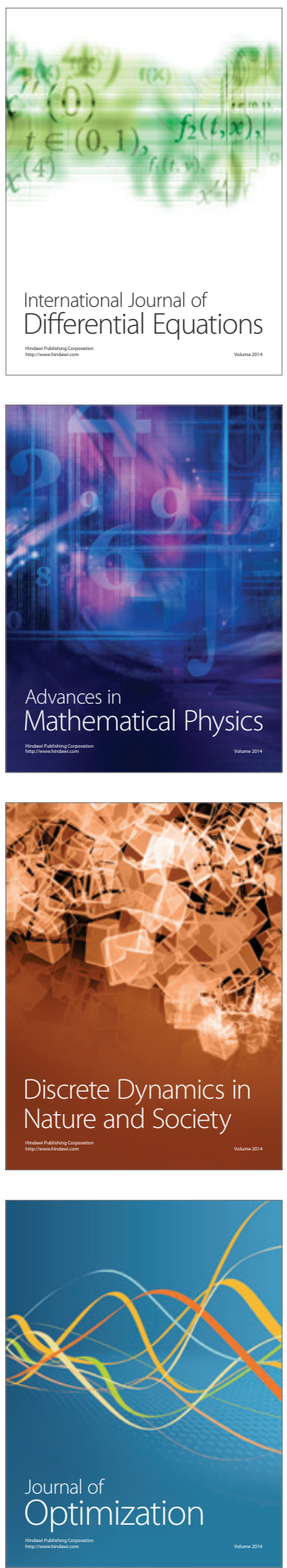